Editorial

\title{
Another Journal on Mathematical Logic and Mathematical Physics?
}

\author{
Angel Garrido \\ University National UNED, Department of Fundamental Mathematics, Faculty of Sciences, Paseo \\ Senda del Rey, 9, 28040, Madrid, Spain; E-Mail: agarrido@mat.uned.es; Tel.: +34-91-3987237; \\ Fax: +34-91-3986944
}

Received: 31 August 2011 / Accepted: 31 August 2011 / Published: 1 September 2011

\section{Introduction}

It is my great pleasure to welcome you to Axioms: Mathematical Logic and Mathematical Physics, a new open access journal, which is dedicated to the foundations (structure and axiomatic basis, in particular) of mathematical and physical theories, not only on crisp or strictly classical sense, but also on fuzzy and generalized sense. This includes the more innovative current scientific trends, devoted to discover and solving new, defying problems.

Our new journal does not try to be the same as those journals already dedicated to this field. Below we highlight what makes Axioms: Mathematical Logic and Mathematical Physics different.

Axioms: Mathematical Logic and Mathematical Physics (ISSN 2075-1680) is an international, open access journal which provides an advanced forum for studies related to axioms. It publishes reviews, regular research papers and short communications. Our aim is to encourage scientists to publish their results in as much detail as possible. There is no restriction on the length of papers. The principal features of this journal will be:

- manuscripts regarding research proposals and research ideas will be particularly welcomed,

- manuscripts concerning summaries and surveys on research cooperation and projects to give information for a broad field of scientists and readers,

The prime goal of this journal, Axioms: Mathematical Logic and Mathematical Physics, is to publish first-class, original research articles under an open access policy with minimal fees for the authors. 
The quality of the published articles will be assured by a fast yet rigorous peer-review process. For this reason, we have selected the members of the Editorial Board with the criterion to "select only the best people".

\section{Essentials}

Since Axioms: Mathematical Logic and Mathematical Physics is published as an online journal there are no page restrictions in place.

However, we distinguish between different types of publications:

- Short Communications contain key elements of a larger research project or preliminary results, and will be published rapidly.

- Full Articles are comprehensive reports on original research of the highest quality.

- Finally, Ideas will discuss novel and thought-provoking scientific ideas and concepts that do not necessarily have to be fully proven (yet).

- In our Book Review section, we will publish helpful reviews on new Mathematical Logic and Mathematical Physics related books.

Again the overarching aim of Axioms: Mathematical Logic and Mathematical Physics is to publish excellent, peer-reviewed articles.

The scope of Axioms: Mathematical Logic and Mathematical Physics embraces many scientific disciplines, as long as mathematical logic and mathematical physics are a crucial part of the scientific research. More specifically, our journal publishes papers in the areas of:

- axiomatic theories in physics and in mathematics (for example, axiomatic theory of thermodynamics, and also either the axiomatic classical set theory or the axiomatic fuzzy set theory)

- axiomatization, axiomatic methods, theorems, mathematical proofs

- algebraic structures, field theory, group theory

- mathematical analysis

- mathematical logic, and non-classical logics, such as fuzzy logic, modal logic, non-monotonic logic. etc.

- automata theory

- mathematical problems of artificial intelligence

- reasoning under uncertainty

- information theory

- symmetry

- entropy

- graph theory

- complex networks from a mathematical viewpoint

- systems theory

- differential equations and dynamical systems

- classical measure theory, generalized measure theory (in particular, fuzzy measure theory)

- theory of probability and Bayesian inference

- mathematical physics 
- relativity and quantum theories

- mathematical chemistry

- history and philosophy of mathematical thinking

- history and philosophy of physical thinking

\section{Conclusion}

To raise interest in this new journal and to set and maintain the level of quality of the papers, we have carefully selected the members of the Editorial Board, with the aforementioned criterion of "only the best", in the areas of physics, mathematics, as well as the science of computation-all of them very interconnected such that they often overlap, precisely in the most fruitful and unexplored zones. Thank you very much to all the Editorial Board members-and also to the very efficient members of MDPI-for their generous support and collaboration. It is a pride and an honor to be able to work with all of you.

I hope that this exciting project sparks your interest, and that you enjoy reading Axioms: Mathematical Logic and Mathematical Physics. We are looking forward to receiving some of your very best manuscripts for publication in this journal!

(C) 2011 by the authors; licensee MDPI, Basel, Switzerland. This article is an open access article distributed under the terms and conditions of the Creative Commons Attribution license (http://creativecommons.org/licenses/by/3.0/). 